\title{
Global Asymptotic Stability Analysis of Predator-Prey System
}

\author{
Ahmed Buseri Ashine \\ Department of Mathematics, Madda Walabu University, Bale Robe, Ethiopia
}

Email address:

amebuseri@gmail.com

\section{To cite this article:}

Ahmed Buseri Ashine. Global Asymptotic Stability of Predator-Prey System. Mathematical Modelling and Applications. Vol. 1, No. 4, 2017, pp. 40-42. doi: 10.11648/j.mma.20170204.11

Received: May 16, 2017; Accepted: August 24, 2017; Published: September 26, 2017

\begin{abstract}
In this paper, a predator-prey model with Holling type II response function is proposed and analyzed. The model is characterized by a couple of system of first order non-linear differential equations. The objective of the work is to offer mathematical analysis of such model. The equilibrium points are computed, boundedness and criteria for stability and persistent of the system are obtained.
\end{abstract}

Keywords: Prey-Predator, Stability, Persistent, Limit Cycle

\section{Introduction}

The dynamic relation between prey and predator has been studied extensively in the literature. At first sight, preypredator dynamics may seem very simple mathematically, but they are, in fact very difficult and challenging. The classical Lotka-Volterra model is a first stepping stone in the study of prey-predator dynamics and interactions [1, 16].

There is an extensive literature concerned with the dynamical relationship between predator and prey due to its universal existence and importance. Mathematical modeling provides an effective tool in the study of contemporary population ecology [2, 3, 4]. In population dynamics, the functional response of predator to prey density refers to the change in the density of prey attacked per unit time per predator as the prey density changes [4]. Although the preypredator theory has seen much progress, many long standing mathematical and ecological problems remain open [6 - 12].

Consider the prey-predator system:

$$
\left\{\begin{array}{c}
\frac{d x}{d t}=\operatorname{rx}\left(1-\frac{x}{k}\right)-\frac{\alpha x y}{1+n x} \equiv F(x, y) \\
\frac{d y}{d t}=y\left(-s+\frac{\alpha \delta x}{1+n x}\right) \equiv G(x, y)
\end{array}\right.
$$

where $\mathrm{x}, \mathrm{y}$ denote prey and predator population respectively at any time t, and $r, \mathrm{k}, \mathrm{s}, \alpha, \mathrm{n}$ and $\delta$ are all positive constants. Here $\mathrm{r}$ represents the intrinsic growth rate and $\mathrm{k}$ the carrying capacity of the prey; $\mathrm{s}$ is the death rate of the predator; $\frac{\alpha}{n}$ is the maximum number of prey that can be eaten by each predator in unit time; $\frac{1}{n}$ is the density of prey necessary to achieve one half that rate; $\delta$ is the conversion factor denoting the number of newly born predators for each captured prey. The term $\frac{\alpha n}{(1+n x)}$ denotes the functional response of the predator.

\section{Basic Results}

\subsection{Boundedness of the System}

Theorem 1. All the solutions of system (1) are uniformly bounded.

Proof: Define the function $z=x+(1 / \delta) y)$. Then

$\frac{d z}{d t}=\frac{d x}{d t}+\frac{1}{\delta} \frac{d y}{d t}=r x\left(1-\frac{x}{k}\right)-\frac{\alpha x y}{1+n x}-\frac{s}{\delta} y+\frac{\alpha x y}{1+n x}$

For $\delta>0$, we have $\frac{d z}{d t}+u z \leq \frac{k}{4 r}(u+r)^{2}-\frac{1}{\delta}(s-u)$

Now if we choose $u<s$; then $\frac{k}{4 r}(u+r)^{2}-\frac{1}{\delta}(s-u)$ 
is bounded for all $(x, y) \in \mathfrak{R}_{+}^{2}$. Thus we choose $\eta>0$, such that $\frac{d z}{d t}+u z<\eta$

Applying the theory of differential inequality [13] we obtain:

$$
0<z(x, y)<\frac{\eta}{u}\left(1-e^{-u t}\right)+z(x(0), y(0)) e^{-u t}
$$

Which, up on letting $t \rightarrow \infty$, yields $0<z<\frac{\eta}{u}$. So, we have that all the solutions of system (1) that start $\Re_{+}^{2}$ are confined to the origin $\mathrm{A}$, where

$$
A=\left\{(x, y) \in R_{+}^{2}: z=\frac{\eta}{u}+\varepsilon\right\} \mathcal{E}, \text { for any } \varepsilon>0 .
$$

\subsection{Equilibria}

We now study the existence of equilibria of system (1). Particularly we are interested in the interior or positive equilibrium. To begin with we list all possible equilibria:

1. The trivial equilibrium $E_{0}(0,0)$

2. Equilibrium in the absence of predator $E_{1}(k, 0)$

3. The interior (positive) equilibrium can be obtained by solving the following equations

$$
\begin{gathered}
r-\frac{r x}{k}-\frac{\alpha y}{1+n x}=0 \\
-s+\frac{\alpha \delta x}{1+n x}=0
\end{gathered}
$$

Say $E^{*}\left(x^{*}, y^{*}\right)$, where

$$
x^{*}=\frac{s}{(\delta \alpha-s n)}, \text { and } x^{*}=\frac{r \delta}{k}\left(\frac{k(\delta \alpha-s n)-s}{(\delta \alpha-s n)^{2}}\right)
$$

For the existence of positive equilibrium both $\delta \alpha-s n>$ 0 and $\frac{s}{(\delta \alpha-s n)}<1$ must hold.

\subsection{Stability Analysis}

In this section we will consider the stability properties of the equilibria of (1). Stability of equilibrium points is investigated by finding the Jacobian matrices for each equilibrium points.

Now

$$
J=\left(\begin{array}{ll}
F_{x}(x, y) & F_{y}(x, y) \\
G_{x}(x, y) & G_{y}(x, y)
\end{array}\right)=\left(\begin{array}{cc}
r-\frac{2 r x}{k}-\frac{\alpha y(1+n x)-\alpha n x y}{(1+n x)^{2}} & \frac{\alpha x}{1+n x} \\
\frac{\alpha \delta(1+n x)-\alpha n \delta x}{(1+n x)^{2}} & -s+\frac{\alpha \delta x}{1+n x}
\end{array}\right)
$$

From this we have,

$$
\begin{aligned}
& J\left(E_{0}\right)=\left(\begin{array}{cc}
r & 0 \\
0 & -s
\end{array}\right), \text { and } J\left(E_{1}\right)=\left(\begin{array}{cc}
-r & \frac{-\alpha k r}{r(1+k n)} \\
0 & -s+\frac{\delta \alpha r k}{r(1+k n)}
\end{array}\right), \\
& J\left(E_{1}\right)=\left(\begin{array}{ll}
A & B \\
C & 0
\end{array}\right)
\end{aligned}
$$

Where, $A=r-\frac{2 r}{k} \frac{s}{\delta \alpha-s n}-\frac{r}{k \delta a}(k(\delta \alpha-s n)-s)$

$$
B=-\frac{s}{\delta} \text {, and } C=\frac{r}{k \alpha}(k(\delta \alpha-s n)-s)
$$

The Eigen values of this system are roots of the equation $(r-\lambda)(-\lambda-s)=0$. Therefore, $\mathrm{E}_{0}(0,0)$ is unstable (saddle). $\mathrm{E}_{1}(\mathrm{k}, 0)$ is locally asymptotically stable when $\frac{s}{k(\delta \alpha-n s)}>11$ because, Jacobean matrix of $\mathrm{E}_{1}(\mathrm{k}, 0)$, has negative value if $\frac{\sigma}{k(\delta \alpha-n s)}>1$ and unstable(saddle) when $\frac{s}{k(\delta \alpha-n s)} \leq 1$.

Proposition 1 . When both $\mathrm{E}_{0}(0,0)$, and $\mathrm{E}_{1}(\mathrm{k}, 0)$ are saddle, the system is persistent. See [15].

Theorem 2. Suppose $\operatorname{det}\left(\mathrm{E}_{2}\right)=-\mathrm{BC}$ and $\operatorname{tr}\left(\mathrm{E}_{2}\right)=\mathrm{A}$, then $\mathrm{E}_{2}$ is locally asymptotically stable if $-B C>0$, and $A<0$.

Proof: Now A will be negative if $\frac{s}{k(\delta \alpha-s n)}+\frac{\delta \alpha}{k n(\delta \alpha-s n)}>1$. From this it is clear that if $\frac{s}{k(\delta \alpha-s n)}+\frac{\delta \alpha}{k n(\delta \alpha-s n)}>1$, then $\mathrm{E}_{2}$ is locally asymptotically stable.

Proposition 2. If $\frac{s}{k(\delta \alpha-s n)}+\frac{\delta \alpha}{k n(\delta \alpha-s n)}=1$, then the system enters into Hopf type small amplitude periodic solutions (limit cycles) near $\mathrm{E}_{2}$.

\subsection{Existence of Limit Cycle}

In two dimensions, it is well known for prey-predator systems that the existence and stability of a limit cycle is related to the existence and stability of a positive equilibrium. We assume that a positive equilibrium exists, for otherwise the predator population tends to extinction [14].

If the equilibrium is asymptotically stable, there may exist limit cycles, the innermost of which must be unstable from the inside and the outermost of which must be stable from the outside. If the limit cycles do not exist in this case, the equilibrium is globally asymptotically stable. If the positive equilibrium exists and is unstable, there must occur at least one limit cycle.

In the present subsection, we shall prove that system (1) has unique stable limit cycle, when $\mathrm{E}_{2}$ becomes locally unstable.

Let us consider system (1) in the form

$$
\frac{d x}{d t}=x g(x)-y p(x), x(0)>0
$$

$$
\frac{d y}{d t}=y(-s+q(x)), y(0)>0
$$


Where $g(x)=r\left(1-\frac{x}{k}\right) \quad p(x)=\frac{\alpha x}{1+n x} q(x)=\frac{\delta \alpha x}{1+n x}$. We have the following Lemma regard uniqueness of limit cycle of this system.

Lemma1. Suppose in system

$\frac{d}{d x}\left(\frac{x g^{\prime}(x)+g(x)-x g(x) \frac{p \prime(x)}{p(x)}}{-s+q(x)}\right) \leq 0$ in $0 \leq x<x^{*}$ and $x^{*}<x \leq$

$k$. The system (2) has exactly one limit cycle which is globally asymptotically stable with respect to the set $\left.\left.\{(x, y): x>0, y>0\} \backslash\left\{E_{2}\left(x^{*}, y^{*}\right)\right\}\right)\right\}$

Theorem3. If $\frac{s}{k(\delta \alpha-s n)}+\frac{\delta \alpha}{k n(\delta \alpha-s n)} \leq 1$, then system (1) has exactly one limit cycle which is globally asymptotically stable with respect to the set $\{(x, y): x>0, y>$ $\left.\left.0\} \backslash\left\{E_{2}\left(x^{*}, y^{*}\right)\right\}\right)\right\}$

Proof: This will be equivalent to proving

$$
\frac{d}{d x}\left(\frac{x\left(1-\frac{x}{k}\right)+r\left(1-\frac{x}{k}\right)-r\left(1-\frac{x}{k}\right) \frac{1}{1+n x}}{-s+\frac{\delta \alpha x}{1+n x}}\right) \leq 0
$$

Equivalently, $\frac{d}{d x}\left(\frac{x\left(2 x+\frac{1}{n}-k\right)}{x-\lambda}\right) \geq 0$,

Where $\lambda=\frac{s}{\delta \alpha-n s}$. It is equivalent to proving $(x-\lambda)^{2}+$ $\lambda\left(\frac{k-\frac{1}{n}}{2}\right)-\lambda^{2} \geq 00$ or $\frac{k-\frac{1}{n}}{2} \geq \lambda$

That is if $\frac{s}{k(\delta \alpha-s n)}+\frac{\delta \alpha}{k n(\delta \alpha-s n)} \leq 1$.

The equality holds if and only if $\frac{s}{k(\delta \alpha-s n)}+\frac{\delta \alpha}{k n(\delta \alpha-s n)}=1$. This completes the proof.

Combining all these results, we have the following theorem.

Theorem 4. If $\delta \alpha>n s$, then the positive equilibrium point existsand stable provided:

$$
\frac{s}{k(\delta \alpha-s n)}+\frac{\delta \alpha}{k n(\delta \alpha-s n)}>1 \text { and } \frac{s}{k(\delta \alpha-s n)}<1 ;
$$

and globally stable limit cycle exists when

$$
\frac{s}{k(\delta \alpha-s n)}+\frac{\delta \alpha}{k n(\delta \alpha-s n)} \leq 1
$$

\section{Conclusion}

In this paper we have considered a prey-predator system assuming that the predator response is of Holling type II.

We have given conditions for existence and stability of the equilibria and persistent criteria for the system. We have proved that exactly one stable limit cycle occurs in this system when the positive equilibrium is unstable. This proof also enables us to conclude that local asymptotic stability of the positive equilibrium implies its global asymptotic stability.

\section{References}

[1] Lotka, A.: Elements of Physical Biology. Williams and Wilkins, Baltimore (1925).

[2] N. Apreutesei, A. Ducrot, V. Volpert, Competition of species with intra-specific competition, Math. Model. Nat. Phenom., 3, pp. 1-27, 2008.

[3] H. Malchow, S. Petrovskii, E. Venturino, Spatiotemporal Patterns in Ecology and Epidemiology, Chapman \& Hall/CRC Press, Boca Raton, 2008.

[4] Ahmed Buseri Ashine "study on prey-predator model with predator in disease and harvesting" Global Journal of Science Frontier Research 17(2) pp. 23-28.

[5] S. G. Ruan, D. M. Xiao, Global analysis in a predator-prey system with non-monotonic functional response, SIAM J. Appl. Math., 61, pp. 1445-1472, 2001.

[6] Berryman AA. The origins and evolutions of predator-prey theory. Ecology 1992; 73: 1530-5.

[7] Kuang Y, Freedman HI. Uniqueness of limit cycles in Gausetype predator-prey systems. Math Biosci 1988;88: 67-84.

[8] Kuang Y. Nonuniqueness of limit cycles of Gause-type predator-prey systems. Appl Anal 1988;29:269-87.

[9] Kuang Y. On the location and period of limit cycles in Gausetype predator-prey systems. J Math Anal Appl 1989; 142: 130-43.

[10] Kuang Y. Limit cycles in a chemo stat-related model. SIAM J Appl Math 1989;49: 1759-67.

[11] Kuang Y. Global stability of Gauss-type predator-prey systems. J Math Biol 1990;28: 463-74.

[12] Berreta E, Kuang Y. Convergence results in a well-known delayed predator-prey system. J Math Anal Appl 1996;204: 840-53.

[13] Birkoff $G$, Rota GC, Ordinary Differential equations, Ginn; 982.

[14] Freedman HI. Deterministic mathematical models population ecology. New York: Marcel Dekker; 1980.

[15] Freedman HI. Waltman P. Persistence in models of three interacting predator-prey population. Math Biosci 1984; 68: 213-31.

[16] Volterra, V.: Variazionie fluttauazionidel numero dindividui in species animals conviventii. Mem Acd. Linciei 2, 31-33 (1926). 